\title{
Botrytis spp.: A Contemporary Perspective and Synthesis of Recent Scientific Developments of a Widespread Genus that Threatens Global Food Security
}

\author{
Jonathan K. Richards, ${ }^{1, \dagger}$ Chang-Lin Xiao, ${ }^{2}$ and Wayne M. Jurick II ${ }^{3, \dagger}$ \\ ${ }^{1}$ Assistant Professor, Department of Plant Pathology and Crop Physiology, Louisiana State University Agricultural Center, Baton Rouge, LA \\ ${ }^{2}$ Supervisory Research Plant Pathologist, Commodity Protection and Quality Research Unit, USDA-ARS, Parlier, CA \\ ${ }^{3}$ Lead Scientist and Research Plant Pathologist, Food Quality Laboratory, USDA-ARS, Beltsville, MD \\ Accepted for publication 22 November 2020.
}

ABSTRACT

\begin{abstract}
This perspective presents a synopsis of the topics contained in the Phytopathology Pathogen Spotlight on Botrytis spp. causing gray mold, including pathogen biology and systematics, genomic characterization of new species, perspectives on genome editing, and fungicide resistance. A timely breakthrough to engineer host plant resistance against the gray mold fungus has been demonstrated in planta and may augment chemical controls in the near future. While B. cinerea has garnered much of the research attention, other economically important Botrytis spp. have been identified and characterized via morphological and genomebased approaches. Gray mold control is achieved primarily through fungicide applications but resistance to various chemical classes is a
\end{abstract}

major concern that threatens global plant health and food security. In this issue, new information on molecular mechanism(s) of fungicide resistance and ways to manage control failures are presented. Finally, a significant leap in fundamental pathogen biology has been achieved via development of CRISPR/Cas9 to assess gene function in the fungus which likely will spawn new control mechanisms and facilitate gene discovery studies.

Keywords: antimicrobial or fungicide resistance, disease resistance, emerging species, fungal pathogens, fungicide resistance, gray mold, genomics, host plant resistance

\section{PATHOGEN BIOLOGY}

Gray mold is caused by fungi within the Ascomycete genus Botrytis and are characterized morphologically by a fine white or gray-white mycelium that occurs over the infected, necrotic, or decayed area of the plant host (Spadaro et al. 2020). As the infected area enlarges, the lesion becomes darker while the edges remain pale. Gray spore masses (containing conidiophores bearing conidia) appear during advanced colonization, with some isolates producing highly melanized sclerotia for long-term survival (Holz et al. 2007). In suitable environmental conditions, sclerotia can germinate to produce apothecia or mycelium that give rise to conidiophores bearing conidia, which serve as the primary source of inoculum. While the fungus has the capability to reproduce sexually, apothecia are rarely found in nature but can be readily obtained in the laboratory (Faretra et al. 1988). Symptoms vary among pathosystems involving Botrytis spp. and include both pre- and postharvest diseases. However, a unifying characteristic is the gray mycelium produced by Botrytis spp. on many different hosts including fruits, vegetables, and ornamental plants.

The genus Botrytis consists of $\sim 35$ necrotrophic species, some having a very broad host range (e.g., B. cinerea and B. pseudocinerea) impacting $\sim 1,400$ different plant species (Elad et al. 2016). Economically important examples include tomato, pepper, grape, strawberry, raspberry, apple, pear, chickpea, and cut flowers, among others. Conversely, other species have a narrow host range or are even host-specific, including $B$. fabae

†Corresponding authors: J. Richards; JRichards@agcenter.lsu.edu, and W. M. Jurick II; wayne.jurick@usda.gov

Mention of trade names or commercial products in this publication is solely for providing specific information and does not imply recommendation or endorsement by the U.S. Department of Agriculture.

The author(s) declare no conflict of interest

(C) 2021 The American Phytopathological Society (broad bean) and B. calthae (marsh marigold) (Plesken et al. 2015). In some cases, multiple Botrytis species share the same host, e.g., B. squamosa, B. allii, and B. aclada all pose significant economic threats to commercial onion production. Interestingly, B. squamosa is family-specific and pathogenic on onion, garlic, and leek (Allium spp.), while the closely related sister species are restricted to lily (B. elliptica) and daylily (B. deweyae) (Steentjes et al. 2021). Understanding the biological and genetic mechanisms that govern host range and pathogen specificity are key to solving problems incited by Botrytis spp. Work presented by Steentjes et al. (2021) in this issue makes significant contributions to unraveling the molecular and biological underpinnings that dictate the breadth, depth, and in some cases narrow host range of various Botrytis spp. using contemporary molecular and genomics tools.

Diversity within Botrytis spp. is exhibited by cultural morphology, virulence, and host range/specificity. Nevertheless, the unifying theme among all gray mold fungi is their necrotrophic lifestyle in which they kill host cells via secretion of effector proteins to induce cell death, obtain nutrients, and subsequently colonize dead plant tissue (Steentjes et al. 2021; Veloso and van Kan 2018). Interestingly, all Botrytis spp. possess approximately 125 predicted effector genes. In the case of B. squamosa, culture filtrates induce an onion-specific programmed cell death upon leaf infiltration (Steentjes et al. 2021). Unfortunately, the bioinformatically predicted effector repertoire did not reveal any obvious key determinants that distinguish the host specificity of B. squamosa from B. elliptica and B. deweyae, which are closely related species with different host specificities (Valero-Jiménez et al. 2019). However, it is possible through functional genetic studies that the role of these effectors will be determined. It is envisioned that through a combination of functional, genetic, and genomic studies the fungal effectorinteracting partners will be discovered in the host. Translative potential of these findings are realized as new targets for disease control and screening tools for breeders for this impactful group of plant pathogens. 


\section{DISEASE MANAGEMENT}

Host plant resistance is a central component to augment chemical and biological control as part of an integrated disease management system (Stout and Davis 2009). While resistance is currently employed and successfully utilized in various crops (e.g., wheat rusts, cucurbit powdery mildew, and potato late blight), incorporation of gray mold resistance into crop plants has not been realized at a commercial level. This is in part due to the complexity of the overlapping multiple mechanisms governing resistance to gray mold that involve pathogen perception, signaling, response, and metabolite detoxification (AbuQamar et al. 2016). However, incorporation of genetic sources of resistance against Botrytis spp. would provide a crucial biological foundation for crop improvement within the family Solanaceae, especially in tomato (Smith et al. 2014). Gong et al. (2021) in this issue implement a transgenic approach in tobacco to confer resistance to $B$. cinerea as well as to leaf mold caused by Cladosporium fulvum and to salt stress. This was accomplished by overexpression of the endogenous tomato atpA gene (cpATPase CF1 alpha subunit gene) in tobacco. They showed that overexpression of atpA positively regulated plant resistance to $B$. cinerea, balanced reactive oxygen species levels, reduced oxidative damage, and increased photosynthetic efficiency compared with wild-type plants Gong et al. (2021). It is now possible to engineer tomato and extend this broad-spectrum protection to other solanaceous crops to confer resistance to gray mold and other pathogens as a new tool to aid disease management.

Management of diseases caused by Botrytis spp. has been largely dependent on the use of fungicides, and active ingredients with different modes of action have been developed and registered for gray mold control on various crops (Fillinger and Walker 2016; Leroux 2007). The major groups of fungicides effective against Botrytis spp. include methyl-benzimidazole carbamates (MBCs), dicarboximides (DCF), anilinopyrimidines, phenylpyrroles (PPs), KetoReductase inhibitors, quinone outside inhibitors (QoIs), succinate dehydrogenase inhibitors (SDHIs), and multisite toxicants (Fillinger and Walker 2016; Fungicide Resistance Action Committee 2020). However, fungicide resistance in $B$. cinerea populations can often result in control failures (Leroux 2007). The intrinsic characteristics of both pathogen and fungicide contribute to the risk of developing fungicide resistance in fungal pathogens, and $B$. cinerea is considered a high-risk pathogen in this context because of its genetic diversity, short life cycle, and prolific reproduction (Brent and Hollomon 2007). Most modern fungicides that are effective against Botrytis at a low dosage have single-site activity targeting essential cellular functions (Fillinger and Walker 2016), and most of the single-site fungicides are considered medium to high or high-risk fungicides in terms of fungicide resistance development (Fungicide Resistance Action Committee 2020). In this issue, Shao et al. (2021) provide an overview of the current status of resistance to MBCs, DCFs, QoIs, PPs, and SDHIs in $B$. cinerea, particularly in China. One recent trend in fungicide resistance in $B$. cinerea populations is that multiple resistance to more than two groups of chemically unrelated fungicides has become common, and simultaneous multiple resistance to up to seven different classes of fungicides has been reported in various regions (Bardas et al. 2010; Fernández-Ortuño et al. 2014; Rupp et al. 2017; Saito et al. 2016; Shao et al. 2021).

Management of fungicide resistance has become an integral part of control of Botrytis-incited diseases, and detection and monitoring of fungicide resistance in $B$. cinerea populations is important for the development and implementation of resistance management strategies (Fillinger and Walker 2016; Shao et al. 2021). Understanding molecular mechanisms and identification of point mutations conferring resistance is essential to the development of molecular detection tools for monitoring fungicide resistance. In this issue, Shao et al. (2021) discusses recent advances in understanding molecular mechanisms conferring resistance to the main fungicide classes currently used for gray mold control. Among the common single-site fungicides, the PP fungicide fludioxonil has been a very important tool for management of Botrytis diseases because of either no resistance or limited resistance development (Kilani and Fillinger 2016). However, high fludioxonil resistance has been reported recently, although at a low frequency (Sang et al. 2018). Molecular mechanisms of fludioxonil resistance have not been fully understood (Fillinger and Walker 2016). In this issue, Dowling et al. (2021) report that high fludioxonil resistance may be associated with the HOG1 MAPK pathway, and isolates highly resistant to fludioxonil exhibited fitness penalties such as suppressed mycelial growth and sporulation.

The increasing prevalence of multiple resistance in $B$. cinerea populations has limited the options of implementing fungicide rotation or mixture strategies for disease control and management of fungicide resistance (Fernández-Ortuño et al. 2014; Shao et al. 2021). Although novel mode-of-action fungicides may be developed in the future for gray mold control, given the nature of evolving resistance development in $B$. cinerea to fungicides, integrated management strategies combining various approaches such as novel fungicides, induced host resistance, biocontrol, and cultural practices would need to be implemented for both disease suppression and management of fungicide resistance in $B$. cinerea (Elad 2016; Shao et al. 2021).

\section{MOLECULAR PLANT-BOTRYTIS INTERACTIONS}

As necrotrophic pathogens, Botrytis spp. induce necrosis to facilitate host colonization. A significant body of research has described the specific molecules utilized by these organisms that contribute to pathogen virulence and elicit programmed cell death. The $\beta$-1, 4-endoxylanase BcXyn11A is a secreted protein produced by $B$. cinerea and was observed to elicit necrosis (Brito et al. 2006; Noda et al. 2010). Interestingly, although BcXyn11A possesses xylanase activity, the ability of BcXyn11A to induce necrosis was independent of enzymatic activity, indicating that the host recognition of BcXyn11A and not xylan oligomers leads to cell death (Noda et al. 2010). Furthermore, a 25 amino acid peptide derived from BcXyn11A was recently found to be sufficient to elicit cell death, as well as the hallmarks of the plant defense response similar to treatment with native BcXyn11A (Frías et al. 2019). Endopolygalacturonases have also been demonstrated to elicit necrosis and in the cases of Bcpg1 and Bcpg2, may contribute to virulence (Kars et al. 2005; ten Have et al. 1998). In contrast to $\mathrm{BcXyn} 11 \mathrm{~A}$, the enzymatic activity of $\mathrm{Bcpg} 2$ is required to induce host necrosis (Kars et al. 2005). It was therefore hypothesized that host recognition of this protein does not influence the formation of necrosis, but that necrosis is solely due to degradation and loss of cell wall integrity. A gene encoding a cerato-platanin protein, $B c s p l 1$, was found to be highly expressed during infection by $B$. cinerea and the Bcspl1 protein induced a dose-dependent necrosis (Frías et al. 2011). The deletion of this gene resulted in a reduction in virulence, thereby supporting its proposed role as a virulence factor. Necrosis inducing Nep1-like proteins have also been characterized from B. elliptica and B. cinerea (Cuesta Arenas et al. 2010; Staats et al. 2007b). However, despite their ability to induce cell death, they do not appear to significantly impact pathogen virulence. Collectively, these studies have illustrated diverse mechanisms in which Botrytis spp. induce cell death to facilitate its necrotrophic lifestyle. In this issue, Steentjes et al. (2021) discuss the observation that infiltration of $B$. squamosa culture filtrates elicits a host-specific cell death response in onion. They posit that secreted effector proteins may be involved in the induction of host cell death. Additionally, Steentjes et al. (2021) note that $B$. squamosa harbors a multitude of cell wall degrading enzymes that likely play a role in infection. Also in this issue, $\mathrm{Wu}$ et al. (2021) discuss effector gene prediction in B. fragariae. A total of 1,184 secreted proteins were predicted including 154 that are 
classified as putative effectors. It was also noted that $B$. fragariae harbored a significantly larger proportion of species-specific effectors when compared with $B$. cinerea, B. paeoniae, and $B$. tulipae, possibly contributing to its specific interaction with strawberry. The potential necrosis-inducing factors in these systems have yet to be identified, but their discovery will undoubtedly advance our understanding of the mechanisms Botrytis spp. use to elicit cell death.

Small RNAs (sRNAs) are short, 21 to 24 nucleotide RNA molecules that target genes for silencing by association with Argonaute proteins, which subsequently guide the RNA-induced silencing complex. A seminal paper by Weiberg et al. (2013) described the identification of sRNAs in $B$. cinerea that exhibit cross-kingdom gene silencing capabilities. sRNA sequencing revealed B. cinerea sRNAs that were predicted to target and silence Arabidopsis genes involved in the immune response, including MAP kinases, a wall-associated kinase, and a peroxiredoxin. Expression of these host genes was suppressed following $B$. cinerea infection, as well as following coexpression of sRNA candidates and the respective predicted targets in Nicotiana benthamiana. Furthermore, overexpression of the sRNA candidates in Arabidopsis significantly suppressed target gene expression and increased host susceptibility to $B$. cinerea, suggesting the role of these host genes in the defense response (Weiberg et al. 2013). Similarly, an additional sRNA, BcsiR37, was identified and predicted to silence 15 genes in Arabidopsis, including components of the plant immune response (Wang et al. 2017). Taken together, results from recent studies highlight the role of sRNAs produced by $B$. cinerea as virulence factors and their function in cross-kingdom RNAi. The observation that sRNAs may be derived from repetitive loci, as seen with $B$. cinerea $\mathrm{sRNAs}$ siR3 and siR5 existing in multiple copies of long terminal repeat elements, makes functional validation of these loci through deletion problematic (Weiberg et al. 2013). However, the development of a CRISPR/Cas approach as discussed by Hahn and Scalliet (2021) in this issue has the potential to address this limitation by simultaneously editing repetitive loci.

\section{GENOMICS OF BOTRYTIS SPP.}

Advancements in genome sequencing technology have enabled the generation of high-quality contiguous reference genomes for many fungal species of interest. These genomic resources have the potential to expedite gene function research. Recently, a gapless reference genome of $B$. cinerea strain B05.10 was developed using a robust combination of single molecule real-time long read sequencing, short-read sequencing, and the inclusion of a genetic and optical map (Van Kan et al. 2017). The resulting genome assembly comprised 18 chromosomes totaling $42.6 \mathrm{Mb}$ in size and contained 11,701 annotated genes. The utility of this foundational resource was demonstrated by genetic and physical mapping of resistance to two fungicides, boscalid and iprodione, as well as examination of genome-wide recombination hotspots (Van Kan et al. 2017). Annotated reference genomes have also been generated for additional Botrytis spp., including B. calthae, B. convolute, B. elliptica, B. galanthina, B. hyacinthi, B. narcissicola, B. paeoniae, B. porri, and B. tulipae (Valero-Jiménez et al. 2019). Additionally, as highlighted in this issue, Wu et al. (2021) describe the construction of a high-quality reference genome of the strawberry pathogen $B$. fragariae. The use of long-read sequencing resulted in the assembly of the $B$. fragariae genome into 28 supercontigs with a total size of $41.92 \mathrm{Mb}$. These and ongoing sequencing efforts produce critical tools that aid functional validation of genes, such as those implicated in Botrytis spp. pathogenicity or virulence, fungicide resistance, as well as basic biological processes and host adaptation.

Host range differs greatly among Botrytis spp., from extremely broad to a single host. The question remains as to what genes play a role in host specialization. The establishment of a gold standard reference genome of $B$. cinerea, as well as genomic resources for other species within the genus, has enabled comparative genomics analyses to begin answering this question. Valero-Jiménez et al. (2019) recently characterized the secretome of 10 Botrytis spp., revealing an abundance of secreted proteins with hydrolase, oxidoreductase, or isomerase activities. Additionally, previously characterized virulence factors, such as Bcpg1, Bcpg2, and Bcxyn 11A, were identified in all species examined indicating to some extent the presence of shared infection strategies across taxa (Valero-Jiménez et al. 2019; Brito et al. 2006; Kars et al. 2005; ten Have et al. 1998). Although the gene ontologies of predicted secreted proteins were relatively consistent across species, ortholog clustering analyses revealed orthologous groups that were shared among a subset of species or unique to a given species, including species-specific effectors. Similarly, secondary metabolite gene clusters displayed varying degrees of presence or absence among species (Valero-Jiménez et al. 2019). Taken together, these results indicate that Botrytis spp. share common components likely involved in the infection process, yet the level of variation observed suggests specific secreted proteins or secondary metabolite gene clusters may have arisen due to host adaptation. As more representative species are sequenced and newly assembled genomes, such as that of $B$. fragariae presented in this issue (Wu et al. 2021), are incorporated into these comparative analyses, the phenomena of host adaptation can be dissected further by experimental validation.

Genetic transformation is key for the proper validation of gene function. CRISPR/Cas technology has revolutionized functional validation by the ability to introduce specific base edits and has been applied in several plant pathogenic fungi (Gosavi et al. 2020). In this issue, Hahn and Scalliet (2021) provide a perspective on their development and implementation of a marker-free CRISPR/Cas gene editing method in $B$. cinerea that overcomes the necessity of genome-integrated selection markers (Leisen et al. 2020). This new method can be used to make precise edits in genes of interest, as demonstrated by the replacement of codon 272 of the SDHI resistance gene $s d h B$ with codons encoding all possible 20 amino acids. Future directions and applications of this method are also discussed by Hahn and Scalliet (2021), including the direct modification of endogenous gene loci for fluorescent tagging, modification of sRNAs, and development of large-scale mutant collections for functional screens. This method has the potential to be applied to other fungal species, and when combined with the expanding Botrytis spp. genomic resources, will greatly expedite the functional validation of genes.

\section{CLASSIFICATION AND EMERGING BOTRYTIS SPP.}

B. cinerea is the type species of the genus Botrytis. Traditionally, speciation of Botrytis spp. has been based on morphological and cultural characteristics. The most conspicuous morphological characteristics of Botrytis spp. are their conidiophores bearing clusters of conidia that resemble clusters of grapes. The size and shape of conidia and conidiophores; production, organization, and size of sclerotia; and colony morphology on culture media have been commonly used as criteria in speciation of Botrytis (Jarvis 1977, 1980; Saito et al. 2016; Zhou et al. 2014). However, morphological characteristics such as size and shape of conidia may be similar or indistinguishable among species, and colony morphology on media may also depend on the growing conditions (Martinez et al. 2003; Walker et al. 2011), limiting the reliability in the use of morphological characters for Botrytis speciation.

More recently, informative protein-coding genes such as glyceraldehyde-3-phosate dehydrogenase $(G 3 P D H)$, heat-shock protein 60 (HSP60), DNA-dependent RNA polymerase subunit II (RPB2), and necrosis and ethylene-inducing proteins 1 and 2 (NEP1 and NEP2) have been identified and utilized in molecular assays to improve the phylogeny of Botrytis spp., identify new species, and further delineate Botrytis species into separate, closely related 
species or cryptic species (Staats et al. 2005, 2007a; Walker 2016). In this issue, Garfinkel (2021) provides a review of the history of Botrytis speciation. The author discusses the usefulness and limitations of traditional morphological species recognition and biological species recognition for Botrytis speciation and provides an update on recent advancements in molecular phylogenetic analysis for genealogical concordance phylogenetic species recognition for Botrytis speciation. Based on phylogenetic analysis of commonly used protein-coding genes since the work of Staats et al. $(2005,2007 a)$, various new species of Botrytis have been recognized and established and are summarized by Garfinkel (2021). The author also discusses what criteria need to be examined in the description of new species of Botrytis given the diversity within and among Botrytis species. These criteria will be helpful for the Botrytis research community in establishing consensus guidelines used for Botrytis speciation.

\section{SUMMARY}

Botrytis is a fascinating fungal genus with broad-reaching economic and societal impacts, is a main contributor to food waste and loss, and threatens global food security. Solving the gray mold problem on the wide variety of hosts and for the wide range of diseases caused by the pathogen requires a systems-based approach that combines applied, basic, and translational biology. While omics-based studies have added much insight into the mechanisms of fungal virulence, fungicide resistance, and host plant resistance, many secrets of Botrytis at the fundamental level are yet to be discovered and translated into tangible, practical controls and products. Judging from the advances presented in this Pathogen Spotlight, more cutting-edge and next-generation tools, technologies, and approaches are close on the horizon.

\section{ACKNOWLEDGMENTS}

W. M. Jurick II recognizes Gene Lester and Tim Widmer, National Program Leaders in NP 306 Quality and Utilization of Agricultural Products and NP 303 Plant Disease at USDA-ARS for valuing, supporting, and promoting applied and fundamental research to abate food waste and increase food security.

\section{LITERATURE CITED}

AbuQamar, S. F., Moustafa, K., and Tran, L. S. 2016. 'Omics' and plant responses to Botrytis cinerea. Front. Plant Sci. 7:1658.

Bardas, G. A., Veloukas, T., Koutita, O., and Karaoglanidis, G. S. 2010. Multiple resistance of Botrytis cinerea from kiwifruit to SDHIs, QoIs and fungicides of other chemical groups. Pest Manag. Sci. 66:967-973.

Brent, K. J., and Hollomon, D. W. 2007. Fungicide Resistance: The Assessment of Risk. 2nd revised version. Fungicide Resistance Action Committee (FRAC) Monograph No. 2, Brussels.

Brito, N., Espino, J. J., and González, C. 2006. The endo- $\beta-1,4-x y l a n a s e$ Xyn11A is required for virulence in Botrytis cinerea. Mol. Plant-Microbe Interact. 19:25-32.

Cuesta Arenas, Y., Kalkman, E. R. I. C., Schouten, A., Dieho, M., Vredenbregt, P., Uwumukiza, B., Ruiz, M. O., and van Kan, J. A. L. 2010. Functional analysis and mode of action of phytotoxic Nep1-like proteins of Botrytis cinerea. Physiol. Mol. Plant Pathol. 74:376-386.

Dowling, M., Gelain, J., May De Mio, L. L., and Schnabel, G. 2021. Characterization of high fludioxonil resistance in Botrytis cinerea isolates from calibrachoa flowers. Phytopathology 111:478-484.

Elad, Y. 2016. Cultural and integrated control of Botrytis spp. Pages 149-164 in: Botrytis-The Fungus, the Pathogen and its Management in Agricultural Systems. S. Fillinger and Y. Elad, eds. Springer, Cham.

Elad, Y., Pertot, I., Prado, A. M. C., and Stewart, A. 2016. Plant hosts of Botrytis spp. Pages 413-486 in: Botrytis-The Fungus, the Pathogen and its Management in Agricultural Systems. S. Fillinger and Y. Elad, eds. Springer, Cham.

Faretra, F., Antonacci, E., and Pollastro, S. 1988. Sexual-behavior and mating system of Botryotinia fuckeliana, teleomorph of Botrytis cinerea. J. Gen. Microbiol. 134:2543-2550.

Fernández-Ortuño, D., Grabke, A., Li, X., and Schnabel, G. 2014. Independent emergence of resistance to seven chemical classes of fungicides in Botrytis cinerea. Phytopathology 105:424-432.
Fillinger, S., and Walker, A.-S. 2016. Chemical control and resistance management of Botrytis diseases. Pages 189-217 in: Botrytis-The Fungus, the Pathogen and its Management in Agricultural Systems. S. Fillinger and Y. Elad, eds. Springer, Cham.

Frías, M., González, C., and Brito, N. 2011. BcSpl1, a cerato-platanin family protein, contributes to Botrytis cinerea virulence and elicits the hypersensitive response in the host. New Phytol. 192:483-495.

Frías, M., González, M., González, C., and Brito, N. 2019. A 25-residue peptide from Botrytis cinerea xylanase bcxyn11a elicits plant defenses. Front. Plant Sci. 10:474.

Fungicide Resistance Action Committee. 2020. FRAC Code List @2020: Fungal control agents sorted by cross resistance pattern and mode of action (including FRAC Code numbering) Fungicide Resistance Action Committee, Brussels. https://www.frac.info

Garfinkel, A. R. 2021. The history of Botrytis taxonomy, the rise of phylogenetics, and implications for species recognition. Phytopathology 111: 437-454.

Gong, C., Cheng, M., Li, J., Chen, H., Zhang, Z., Qi, H., Zhang, Y., Liu, J., Chen, X. L., and Wang, A. X. 2021. The $\alpha$-subunit of the chloroplast ATP synthase of tomato reinforces the resistance to gray mold and broad-spectrum resistance in transgenic tobacco. Phytopathology 111: 485-495.

Gosavi, G., Yan, F., Ren, B., Kuang, Y., Yan, D., Zhou, X., and Zhou, H. 2020. Applications of CRISPR technology in studying plant-pathogen interactions: Overview and perspective. Phytopathol. Res. 2:21.

Hahn, M., and Scalliet, G. 2021. One cut to change them all: CRISPR/Cas, a groundbreaking tool for genome editing in Botrytis cinerea and other fungal plant pathogens. Phytopathology 111:474-477.

Holz, G., Cortze, S., and Williamson, B. 2007. The ecology of Botrytis on plant surfaces. Pages 9-27 in: Botrytis: Biology, Pathology, and Control. Y. Elad, M. Williamson, P. Tudzynski, and N. Delen, eds. Springer, Dordrecht, The Netherlands.

Jarvis, W. R. 1977. Botryotinia and Botrytis species: Taxonomy, physiology and pathogenicity. A guide to the literature. Monograph No. 15. Can. Dept. Agric.

Jarvis, W. R. 1980. Taxonomy. Pages 1-18 in: The Biology of Botrytis. J. R. Coley-Smith, K. Verhoeff, and W. R. Jarvis, eds. Academic Press,Cambridge, MA.

Kars, I., Krooshof, G. H., Wagemakers, L., Joosten, R., Benen, J. A. E., and Van Kan, J. A. L. 2005. Necrotizing activity of five Botrytis cinerea endopolygalacturonases produced in Pichia pastoris. Plant J. 43:213-225.

Kilani, J., and Fillinger, S. 2016. Phenylpyrroles: 30 years, two molecules and (nearly) no resistance. Front. Microbiol. 7:2014.

Leisen, T., Bietz, F., Werner, J., Wegner, A., Schaffrath, U., Scheuring, D., Willmund, F., Mosbach, A., Scalliet, G., and Hahn, M. 2020. CRISPR/Cas with ribonucleoprotein complexes and transiently selected telomere vectors allows highly efficient marker-free and multiple genome editing in Botrytis cinerea. PLoS Pathog 16:e1008326.

Leroux, L. 2007. Chemical control of Botrytis and its resistance to chemical fungicides. Pages 195-222 in: Botrytis: Biology, Pathology and Control. Y. Elad, ed. Kluwer Academic Publishers, Dordrecht, The Netherlands.

Martinez, F., Blancard, D., Lecomte, P., Levis, C., Dubos, B., and Fermaud, M. 2003. Phenotypic differences between vacuma and transposa subpopulations of Botrytis cinerea. Eur. J. Plant Pathol. 109:479-488.

Noda, J., Brito, N., and González, C. 2010. The Botrytis cinerea xylanase Xyn11A contributes to virulence with its necrotizing activity, not with its catalytic activity. BMC Plant Biol. 10:38.

Plesken, C., Weber, R. W. S., Rupp, S., Leroch, M., and Hahn, M. 2015. Botrytis pseudocinerea is a significant pathogen of several crop plants but susceptible to displacement by fungicide-resistant B. cinerea strains. Appl. Environ. Microbiol. 81:7048-7056.

Rupp, S., Weber, R. W. S., Rieger, D., Detzel, P., and Hahn, M. 2017. Spread of Botrytis cinerea strains with multiple fungicide resistance in German horticulture. Front. Microbiol. 7.

Saito, S., Margosan, D., Michailides, T. J., and Xiao, C. L. 2016. Botrytis californica, a new cryptic species in the $B$. cinerea species complex causing gray mold in blueberries and table grapes. Mycologia 108:330-343.

Sang, C., Ren, W., Wang, J., Xu, H., Zhang, Z., Zhou, M., Chen, C. J., and Wang, K. 2018. Detection and fitness comparison of target-based highly fludioxonil-resistant isolates of Botrytis cinerea from strawberry and cucumber in China. Pestic. Biochem. Physiol. 147:110-118.

Shao, W., Zhao, Y., and Ma, Z. 2021. Advances in understanding fungicide resistance in Botrytis cinerea in China. Phytopathology 111:455-463.

Smith, J. E., Mengesha, B., Tang, H., Mengiste, T., and Bluhm, B. 2014. Resistance to Botrytis cinerea in Solanum lycopersicoides involves widespread transcriptional reprogramming. BMC Genomics 15:334.

Spadaro, D., Torres, R., Errampalli, D., Evertt, K., Ramos, L., and Mari, M. 2020. Pome fruits. Pages 55-112 in: Postharvest Pathology of Fresh 
Horticultural Produce. L. Palou and J. L. Smilanick, eds. CRC Press, Boca Raton, FL.

Staats, M., Van Baarlen, P., Schouten, A., and Van Kan, J. A. L. 2007 b. Functional analysis of NLP genes from Botrytis elliptica. Mol. Plant Pathol. 8:209-214

Staats, M., van Baarlen, P., Schouten, A., van Kan, J. A. L., and Bakker, F. T. 2007a. Positive selection in phytotoxic protein-encoding genes of Botrytis species. Fun. Gen. Biol. 44:52-63.

Staats, M., van Baarlen, P., and van Kan, J. A. L. 2005. Molecular phylogeny of the plant pathogenic genus Botrytis and the evolution of host specificity. Mol. Biol. Evol. 22:333-346.

Steentjes, M. B. F., Scholten, O. E., and van Kan, J. A. L. 2021. Peeling the onion: Towards a better understanding of Botrytis diseases of onion. Phytopathology 111:464-473.

Stout, M., and Davis, J. 2009. Keys to the increased use of host plant resistance in integrated pest management. Pages 163-181 in: Integrated Pest Management: Innovation-Development Process. R. Peshin and A. K. Dhawan, eds. Springer, Dordrecht.

ten Have, A., Mulder, W., Visser, J., and Van Kan, J. A. L. 1998. The endopolygalacturonase gene Bcpgl is required to full virulence of Botrytis cinerea. Mol. Plant-Microbe Interact. 11:1009-1016.

Valero-Jiménez, C. A., Veloso, J., Staats, M., and Van Kan, J. A. L. 2019. Comparative genomics of plant pathogenic Botrytis species with distinct host specificity. BMC Genomics 20:203.

Van Kan, J. A. L., Stassen, J. H. M., Mosbach, A., Van Der Lee, T. A. J., Faino, L., Farmer, A. D., Papasotiriou, D. G., Zhou, S., Seidl, M. F., Cottam, E.,
Edel, D., Hahn, M., Schwartz, D. C., Dietrich, R. A., Widdison, S., and Scalliet, G. 2017. A gapless genome sequence of the fungus Botrytis cinerea. Mol. Plant Pathol. 18:75-89.

Veloso, J., and van Kan, J. A. 2018. Many shades of grey in Botrytis-host plant interactions. Trends Plant Sci. 23:613-622.

Walker, A.-S. 2016. Diversity within and between species of Botrytis. Pages 91-125 in: Botrytis-The Fungus, the Pathogen and its Management in Agricultural Systems. S. Fillinger and Y. Elad, eds. Springer, Cham.

Walker, A.-S., Gautier, A., Confais, J., Martinho, D., Viaud, M., Le Pêcheur, P., Dupont, J., and Fournier, E. 2011. Botrytis pseudocinerea, a new cryptic species causing gray mold in French vineyards in sympatry with Botrytis cinerea. Phytopathology 101:1433-1445.

Wang, M., Weiberg, A., Dellota, E., Yamane, D., and Jin, H. 2017. Botrytis small RNA Bc-siR37 suppresses plant defense genes by cross-kingdom RNAi. RNA Biol. 14:421-428.

Weiberg, A., Wang, M., Lin, F. M., Zhao, H., Zhang, Z., Kaloshian, I., Huang, H., and Jin, H. 2013. Fungal small RNAs suppress plant immunity by hijacking host RNA interference pathways. Science 342:118-123.

Wu, Y., Saski, C., Schnabel, G., Xiao, S., and Hu, M. 2021. A high-quality genome resource of Botrytis fragariae, a new and rapidly spreading fungal pathogen causing strawberry gray mold in the United States. Phytopathology 111:496-499.

Zhou, Y. J., Zhang, J., Wang, X. D., Yang, L., Jiang, D. H., Li, G. Q., Hsiang, T., and Zhuang, W. Y. 2014. Morphological and phylogenetic identification of Botrytis sinoviticola, a novel cryptic species causing gray mold disease of table grapes (Vitis vinifera) in China. Mycologia 106:43-56. 